\title{
Global analysis of almost periodic positive solution of a multispecies discrete Gilpin-Ayala mutualism system with feedback controls
}

\author{
Hui Zhang ${ }^{\text {a }}$ and Yingqi Li \\ Mathematics and OR Section Xi'an Research Institute of High-tech HongqingTown,Xi'an, China
}

\begin{abstract}
This paper discusses a multispecies discrete Gilpin-Ayala mutualism system with feedback controls. We firstly obtain the permanence of the system. Assuming that the coefficients in the system are almost periodic positive sequences, we obtain the sufficient conditions for the existence of a unique almost periodic positive solution which is globally attractive.
\end{abstract}

Keywords: Global attractivity; almost periodic solution; discrete; Gilpin-Ayala mutualism system; permanence

\section{Introduction}

As we all known, investigating the almost periodic positive solutions of discrete population dynamics model with feedback controls has more extensively practical application value (see [1-3] and the references cited therein).Wang [1] considered a nonlinear single species discrete system with feedback control

$$
\left\{\begin{array}{l}
N(n+1)=N(n) \text { ex } \operatorname{pr}\left([n)\left(1-\frac{N^{\theta}(n)}{k(n)}-c(n) u(n)\right)\right], \\
\Delta u(k)=-a(n) u(n)+b(n) N^{\delta}(n) .
\end{array}\right.
$$

Some sufficient conditions which assure the unique existence and global attractivity of almost periodic positive solution are obtained.

In this paper, we investigate the dynamic behavior of the following multispecies discrete GilpinAyalamutualism model with feedback controls

$$
\left\{\begin{array}{l}
x_{i}(k+1)=x_{i}(k) \text { ex }\left\{\left\{a_{i}(k)-b_{i}(k)\left(x_{i}(k)\right)^{\theta_{i i}}+\sum_{j=1, j \neq i}^{n} \frac{c_{i j}(k)\left(x_{j}(k)\right)^{\theta_{i j}}}{d_{i j}+\left(x_{j}(k)\right)^{\theta_{i j}}}-e_{i}(k) u_{i}(k)\right\},\right. \\
\Delta u_{i}(k)=-f_{i}(k) u_{i}(k)+\sum_{j=1}^{n} g_{i j}(k) x_{j}(k),
\end{array}\right.
$$

acCorrespondence author: zh53054958@163.com

(C) 2016. The authors - Published by Atlantis Press 
where $i=1,2, \ldots, n ; x_{i}(k)$ stand for the densities of species $x_{i}$ at the $k$ th generation, $a_{i}(k)$ represent the natural growth rates of species $x_{i}$ at the $k$ th generation, $b_{i}(k)$ are the intra specific effects of the $k$ th generation of species $x_{i}$ on own population, and $c_{i j}(k)$ measure the inter specific mutualism effects of the $k$ th generation of species $x_{j}$ on species $x_{i}(i, j=1,2, \ldots, n, i \neq j), d_{i j}$ are positive control constants. $\theta_{i i}$ and $\theta_{i j}$ are positive constants.

Throughout this paper, we assume that:

(H1) $\left\{a_{i}(k)\right\},\left\{b_{i}(k)\right\},\left\{c_{i j}(k)\right\},\left\{e_{i}(k)\right\},\left\{f_{i}(k)\right\}$ and $\left\{g_{i j}(k)\right\}$ are bounded nonnegative almost periodic sequences such

That

$$
\begin{aligned}
& 0<a_{i}^{l} \leq a_{i}(k) \leq a_{i}^{u}{ }_{i}, 0<b_{i}^{l} \leq b_{i}(k) \leq b^{u}{ }_{i}, 0<c_{i j}^{l} \leq c_{i j}(k) \leq c^{u}{ }_{i j}, \\
& 0<e_{i}^{l} \leq e_{i}(k) \leq e_{i}^{u}, 0<f_{i} \leq f_{i}(k) \leq f^{u}{ }_{i}<1,0<g_{i j}^{l} \leq g_{i j}(k) \leq g^{u}{ }_{i j} .
\end{aligned}
$$

From the point of view of biology, we assume that $\mathbf{x}(0)=\left(x_{1}(0), x_{2}(0), \ldots, x_{n}(0), u_{1}(0), u_{2}(0), \ldots\right.$, $\left.u_{n}(0)\right)>\mathbf{0}$. It is easy to see that, for given $\mathbf{x}(0)$, the system (1) has a positive sequence solution $\mathbf{x}(k)$ $=\left(x_{1}(k), x_{2}(k), \ldots, x_{n}(k), u_{1}(k), u_{2}(k), \ldots, u_{n}(k)\right)\left(k \in Z^{+}\right)$passing through $\mathbf{x}(0)$.

\section{Preliminaries}

Now, we present some results which will play an important role in the proof of the main result.

Definition 2.1[4] A sequence $x: \mathrm{Z} \rightarrow \mathrm{R}$ is called an almost periodic sequence if the $\varepsilon$-translation set of $x$

$$
E\{\varepsilon, x\}=\{\tau \in Z:|x(n+\tau)-x(n)|<\varepsilon, \forall n \in Z\}
$$

is a relatively dense set in $\mathrm{Z}$ for all $\varepsilon>0$; that is, for any given $\varepsilon>0$; there exists an integer $l(\varepsilon)>$ 0 such that each interval of length $l(\varepsilon)$ contains an integer $\tau \in E\{\varepsilon, x\}$ with

$$
|x(n+\tau)-x(n)|<\varepsilon, \forall n \in Z \text {. }
$$

$\varepsilon$ is called an $\varepsilon$-translation number of $\mathrm{x}(\mathrm{n})$.

Definition 2.2[5] A sequence $x: \mathrm{Z}^{+} \rightarrow \mathrm{R}$ is called an asymptotically almost periodic sequence if

$$
x(n)=p(n)+q(n), \forall n \in Z^{+},
$$

Where $p(n)$ is an almost periodic sequence and

$$
\lim _{n \rightarrow+\infty} q(n)=0 \text {. }
$$

Theorem2.3[5] $\{x(n)\}$ is an asymptotically almost periodic sequence if and only if, for any sequence $m_{i} \subset Z$ satisfying $m_{i}>0$ and $m_{i} \rightarrow \infty$ as $i \rightarrow \infty$ there exists a subsequence $\left\{m_{i_{k}}\right\} \subset\left\{m_{i}\right\}$ such that the sequence $\left\{x\left(n+m_{i_{k}}\right)\right\}$ converges uniformly for all $n \in \mathrm{Z}^{+}$as $k \rightarrow \infty$.

Theorem2.4 ([6]) Assume that sequence $\{x(n)\}$ satisfies $x(n)>0$ and

$$
\left.x(n+1) \leq x(n) \text { ex } \mathrm{p} a(n)-b(n) x^{\alpha}(n)\right\}
$$

For $n \in N$, where $a(n)$ and $b(n)$ are non-negative sequences bounded above and below by positive constants, $\alpha$ is a positive constant. Then

$$
\limsup _{n \rightarrow+\infty} x(n) \leq\left(\frac{1}{\alpha b^{i}}\right)^{\frac{1}{\alpha}} \exp \left\{a^{u}-\frac{1}{\alpha}\right\} .
$$

Theorem 2.5([7]) Assume that sequence $\{x(n)\}$ satisfies

$$
\begin{gathered}
\left.x(n+1) \geq x(n) \text { ex } \operatorname{pa}(n)-b(n) x^{\alpha}(n)\right\}, n \geq N_{0} \\
\limsup _{n \rightarrow+\infty} x(n) \leq x^{*}
\end{gathered}
$$

and $x\left(N_{0}\right)>0$, where $a(n)$ and $b(n)$ are non-negative sequences bounded above and below by positive constants, $\alpha$ is a positive constant and $N_{0} \in N$. Then

$$
\liminf _{n \rightarrow+\infty} x(n) \geq \min \left\{\left(\frac{a^{l}}{b^{u}}\right)^{\frac{1}{\alpha}} \exp \left\{a^{l}-b^{u}\left(x^{*}\right)^{\alpha}\right\},\left(\frac{a^{l}}{b^{u}}\right)^{\frac{1}{\alpha}}\right\}
$$

Theorem2.6 ([8]) Assume that $A>0$ and $y(0)>1$, and further suppose that

$$
y(n+1) \leq A y(n)+B(n), n=1,2,3, \cdots .
$$


Then for any integer $k \leq n$,

$$
y(n) \leq A^{k} y(n-k)+\sum_{i=0}^{k-1} A^{i} B(n-i-1)
$$

Especially, if $A<1$ and $B$ is bounded above with respect to $M$, then

$$
\limsup _{n \rightarrow \infty} y(n) \leq \frac{M}{1-A}
$$

Theorem2.7 ([8]) Assume that $A>0$ and $y(0)>1$, and further suppose that

Then for any integer $k \leq n$,

$$
y(n+1) \geq A y(n)+B(n), n=1,2,3, \cdots .
$$

$$
y(n) \geq A^{k} y(n-k)+\sum_{i=0}^{k-1} A^{i} B(n-i-1)
$$

Especially, if $A<1$ and $B$ is bounded below with respect to $m$, then

$$
\liminf _{n \rightarrow \infty} y(n) \geq \frac{m}{1-A}
$$

\section{Permanence}

In this section, we establish the permanence result for system (1.1).

Proposition 3.1 Assume that the condition (H1) holds, furthermore,

$$
a_{i}^{l}-e_{i}^{u} N_{i}>0,
$$

Then system (1.1) is permanent, that is, there exist positive constants $m_{i}, M_{i}, n_{i}$ and $N_{i}(i=1,2, \ldots, n)$ which are independent of the solutions of system (1.1), such that for any positive solution $\left(x_{1}(k)\right.$, $\left.x_{2}(k), \ldots, x_{n}(k), u_{1}(k), u_{2}(k), \ldots, u_{n}(k)\right)$ of system (1.1), one has:

$$
\begin{gathered}
m_{i} \leq \liminf _{k \rightarrow+\infty} x_{i}(k) \leq \limsup _{k \rightarrow+\infty} x_{i}(k) \leq M_{i}, \\
n_{i} \leq \liminf _{k \rightarrow+\infty} u_{i}(k) \leq \limsup _{k \rightarrow+\infty} u_{i}(k) \leq N_{i},
\end{gathered}
$$

Where

$$
\begin{gathered}
M_{i}=\frac{1}{b_{i}^{l}} \exp \left\{a_{i}^{u}+\sum_{j=1, j \neq i}^{n} c_{i j}^{u}-1\right\}, \\
m_{i}=\frac{a_{i}^{l}-e_{i}^{u} N_{i}}{2 b_{i}^{u}} \min \left\{1, \exp \left\{a_{i}^{l}-e_{i}^{u} N_{i}-b_{i}^{u} M_{i}\right\}\right\}, \\
N_{i}=\frac{1}{f_{i}^{l}} \sum_{j=1}^{n} g_{i j}^{u} M_{j}, n_{i}=\frac{1}{f_{i}^{u}} \sum_{j=1}^{n} g_{i j}^{l} m_{j} .
\end{gathered}
$$

Proof. The proof of Proposition 3.1 is similar to that of Theorem 3.1 in Ref. [3]. So we omit the detail here.

We denote by $\Omega$ the set of all solutions $\left(x_{1}(k), x_{2}(k), \ldots, x_{n}(k), u_{1}(k), u_{2}(k), \ldots, u_{n}(k)\right)$ of system (1.1) satisfying $m_{i} \leq x_{i}(k) \leq M_{i}, n_{i} \leq u_{i}(k) \leq N_{i}(i=1,2, \ldots, n)$ for all $k \in Z^{+}$.

Proposition 3.2([3]) Assume that the conditions (H1) and (3.1) hold. Then $\Omega \neq \Phi$.

\section{Almost periodic solution}

The main results of this paper concern the global attractivity of almost periodic positive solution of system (1.1)with conditions (H1) and (3.1).

Theorem 4.1 Assume that (H1), (3.1) and

(H2) $\rho_{i}=\max \left\{\left|1-\theta_{i i} b_{i}^{l} m_{i}^{\theta_{i j}}\right|,\left|1-\theta_{i i} b_{i}^{u} M_{i}^{\theta_{i j}}\right|\right\}+\sum_{j=1, j \neq i}^{n} \frac{\theta_{i j} c_{i j}^{u} M_{j}^{\theta_{i j}}}{d_{i j}}+e_{i}^{u}<1$, 


$$
\varphi_{i}=1-f_{i}^{l}+\sum_{j=1}^{n} g_{i j}^{u} M_{j}<1, i=1,2, \cdots, n,
$$

hold. Then any positive solution $\left(x_{1}(k), x_{2}(k), \ldots, x_{n}(k), u_{1}(k), u_{2}(k), \ldots, u_{n}(k)\right)$ of system (1.1) is globally attractive.

Proof. Assume that $\left(p_{1}(k), p_{2}(k), \ldots, p_{n}(k), v_{1}(k), v_{2}(k), \ldots, v_{n}(k)\right)$ is a solution of system (1.1) satisfying (H1) and (3.1). Let

Since

$$
x_{i}(k)=p_{i}(k) \exp \left\{q_{i}(k)\right\}, u_{i}(k)=v_{i}(k)+w_{i}(k) .
$$

$$
\begin{gathered}
\left.q_{i}(k+1)=\ln x_{i}(x+1)-\ln p_{i}(x+1)=q_{i}(k)\left(1-\theta_{i i} b_{i}(k)\left[p_{i}(k) \operatorname{ex~} \lambda_{i}(k) q_{i}(k)\right\}\right]^{\theta_{i i}}\right) \\
+\sum_{j=1, j \neq i}^{n} \frac{d_{i j} \theta_{i j} c_{i j}(k) q_{j}(k)\left[p_{j}(k) \operatorname{ex~} \bar{\lambda}_{j}\left\{(k) q_{j}(k)\right\}\right]^{\theta_{i j}}}{\left[d_{i j}+\left(x_{j}(k)\right)^{\theta_{i j}}\right]\left[d_{i j}+\left(p_{j}(k)\right)^{\theta_{i j}}\right]}-e_{i}(u) w_{i}(k),
\end{gathered}
$$

where $\lambda_{i}(k), \overline{\lambda_{j}}(k) \in(0,1)$.

Similarly, we get

$$
\begin{gathered}
w_{i}(k+1)=u_{i}(k+1)-v_{i}(k+1) \\
\left.\left.=\left(1-f_{i}(u)\right) w_{i}(k)+\sum_{j=1}^{n} g_{i j}(k) p_{j}(k) q_{j}(k) \operatorname{ex~} \xi_{j}(k)\right) q_{j}(k)\right\}, \text { wh } \xi_{i}(k) \in(0,1) .
\end{gathered}
$$

To complete the proof, it suffices to show that

$$
\lim _{k \rightarrow+\infty} q_{i}(k)=0, \lim _{k \rightarrow+\infty} w_{i}(k)=0 .
$$

In view of (H2), we can choose $\varepsilon>0$ such that

$$
\begin{gathered}
\rho_{i}^{\varepsilon}=\max \left\{\left|1-\theta_{i i} b_{i}^{l}\left(m_{i}-\varepsilon\right)^{\theta_{i j}}\right|,\left|1-\theta_{i b} b_{i}^{u}\left(M_{i}+\varepsilon\right)^{\theta_{i j}}\right|\right\}+\sum_{j=1, j \neq i}^{n} \frac{\theta_{i j} c_{i j}^{u}\left(M_{j}+\varepsilon\right)^{\theta_{i j}}}{d_{i j}}+e_{i}^{u}<1, \\
\varphi_{i}^{\varepsilon}=1-f_{i}^{l}+\sum_{j=1}^{n} g_{i j}^{u}\left(M_{j}+\varepsilon\right)<1, i=1,2, \cdots, n .
\end{gathered}
$$

Let $\rho=\max \left\{\rho_{i}^{\varepsilon}, \varphi_{i}^{\varepsilon}\right\}$, then $\rho<1$. According to Proposition 3.1, there exists a positive integer $k_{0} \in Z^{+}$ such that

fork $\geq k_{0}$.

$$
m_{i}-\varepsilon \leq x_{i}(k) \leq M_{i}+\varepsilon, m_{i}-\varepsilon \leq p_{i}(k) \leq M_{i}+\varepsilon,
$$

From (4.1), we get

$$
\begin{gathered}
\left|q_{i}(k+1)\right|=\max \left\{\left|1-\theta_{i i} b_{i}^{l}\left(m_{i}-\varepsilon\right)^{\theta_{i j}}\right|, \mid 1-\theta_{i i} b_{i}^{u}\left(M_{i}+\varepsilon\right)^{\theta_{i j}}\right\} \\
\left|q_{i}(k)\right|+\sum_{j=1, j \neq i}^{n} \frac{\theta_{i j} c_{i j}^{u}\left(M_{j}+\varepsilon\right)^{\theta_{i j}}}{d_{i j}}\left|q_{j}(k)\right|+e_{i}^{u}\left|w_{i}(k)\right|, \\
\left|w_{i}(k+1)\right|=\left(1-f_{i}^{l}\right)\left|w_{i}(k)\right|+\sum_{j=1}^{n} g_{i j}^{u}\left(M_{j}+\varepsilon\right)\left|q_{j}(k)\right|<1,
\end{gathered}
$$

fork $\geq k_{0}$.In view of (4.3), we get

$$
\max \left\{\max _{1 \leq i \leq n}\left|q_{i}(k+1)\right|, \max _{1 \leq i \leq n}\left|w_{i}(k+1)\right|\right\} \leq \rho \max \left\{\max _{1 \leq i \leq n}\left|q_{i}(k)\right|, \max _{1 \leq i \leq n}\left|w_{i}(k)\right|\right\}, \quad k \geq k_{0} .
$$

This implies

$$
\max \left\{\max _{1 \leq i \leq n}\left|q_{i}(k)\right|, \max _{1 \leq i \leq n}\left|w_{i}(k)\right|\right\} \leq \rho^{k-k_{0}} \max \left\{\max _{1 \leq i \leq n}\left|q_{i}\left(k_{0}\right)\right|, \max _{1 \leq i \leq n}\left|w_{i}\left(k_{0}\right)\right|\right\}, \quad k \geq k_{0} .
$$

Then (4.2) holds and we can obtain

$$
\lim _{k \rightarrow+\infty}\left|x_{i}(k)-p_{i}(k)\right|=0, \lim _{k \rightarrow+\infty}\left|u_{i}(k)-v_{i}(k)\right|=0
$$

Therefore, positive solution $\left(x_{1}(k), x_{2}(k), \ldots, x_{n}(k), u_{1}(k), u_{2}(k), \ldots, u_{n}(k)\right)$ of system (1.1) is globally attractive.

Theorem 4.2 Assume that (3.1), (H1) and (H2) hold. Then system (1.1) admits a unique almost periodic positive solution which is globally attractive.

Proof.It follows from Proposition 3.2 that there exists a solution $\left(x_{1}(k), x_{2}(k) \ldots x n(k), u 1(k), u_{2}(k)\right.$, 
$\ldots, u_{n}(k)$ ) of system (1.1) satisfying $m_{i} \leq x_{i}(k) \leq M_{i}, n_{i} \leq u_{i}(k) \leq N_{i}, k \in Z^{+}$. Let $\left\{\delta_{k}\right\}$ be any integer valued sequence such that $\delta_{k} \rightarrow+\infty$ as $k \rightarrow+\infty$. Using the Mean Value Theorem, for $p \neq q$, we get

$$
\begin{aligned}
& \ln x_{i}\left(k+\delta_{p}\right)-\ln x_{i}\left(k+\delta_{q}\right)=\frac{1}{\theta_{i i} \xi_{i}(k, p, q)}\left[\left(x_{i}\left(k+\delta_{p}\right)\right)^{\theta_{i i}}-\left(x_{i}\left(k+\delta_{q}\right)\right)^{\theta_{i i}}\right], \\
& \ln x_{i}\left(k+\delta_{p}\right)-\ln x_{i}\left(k+\delta_{q}\right)=\frac{1}{\eta_{i}(k, p, q)}\left[x_{i}\left(k+\delta_{p}\right)-x_{i}\left(k+\delta_{q}\right)\right]
\end{aligned}
$$

where $\xi_{i}(k, p, q)$ lies between $\left(x_{i}\left(k+\delta_{p}\right)\right)^{\theta_{i i}}$ and $\left(x_{i}\left(k+\delta_{q}\right)\right)^{\theta_{i i}}$, and $\eta_{i}(k, p, q)$ lies between $x_{i}\left(k+\delta_{p}\right)$ and $x_{i}\left(k+\delta_{q}\right)$. Then

$$
\left|\left(x_{i}\left(k+\delta_{p}\right)\right)^{\theta_{i i}}-\left(x_{i}\left(k+\delta_{q}\right)\right)^{\theta_{i i}}\right| \leq \theta_{i i} M_{i}^{\theta_{i i}}\left|\ln x_{i}\left(k+\delta_{p}\right)-\ln x_{i}\left(k+\delta_{q}\right)\right| .
$$

From the first equation of (1.1), we have

$$
\begin{gathered}
\left|\ln x_{i}\left(k+1+\delta_{p}\right)-\ln x_{i}\left(k+1+\delta_{q}\right)\right|=\mid \ln x_{i}\left(k+\delta_{p}\right)-\ln x_{i}\left(k+\delta_{q}\right) \\
+a_{i}\left(k+\delta_{p}\right)-b_{i}\left(k+\delta_{p}\right)\left(x_{i}\left(k+\delta_{p}\right)\right)^{\theta_{i i}}+\sum_{j=1}^{n} c_{i j}\left(k+\delta_{p}\right) \frac{\left(x_{j}\left(k+\delta_{p}\right)\right)^{\theta_{i j}}}{d_{i j}+\left(x_{j}\left(k+\delta_{p}\right)\right)^{\theta_{i j}}} \\
-a_{i}\left(k+\delta_{q}\right)-b_{i}\left(k+\delta_{q}\right)\left(x_{i}\left(k+\delta_{q}\right)\right)^{\theta_{i i}}+\sum_{j=1}^{n} c_{i j}\left(k+\delta_{q}\right) \frac{\left(x_{j}\left(k+\delta_{q}\right)\right)^{\theta_{i j}}}{d_{i j}+\left(x_{j}\left(k+\delta_{q}\right)\right)^{\theta_{i j}}} \\
+\left[e_{i}\left(k+\delta_{q}\right)-e_{i}\left(k+\delta_{p}\right)\right] u\left(k+\delta_{q}\right)-e_{i}\left(k+\delta_{p}\right)\left[u\left(k+\delta_{p}\right)-u\left(k+\delta_{q}\right)\right] \mid \\
\leq\left|\ln x_{i}\left(k+\delta_{p}\right)-\ln x_{i}\left(k+\delta_{q}\right)-b_{i}\left(k+\delta_{p}\right)\left[\left(x_{i}\left(k+\delta_{p}\right)\right)^{\theta_{i i}}-\left(x_{i}\left(k+\delta_{q}\right)\right)^{\theta_{i i}}\right]\right| \\
+\left|a_{i}\left(k+\delta_{p}\right)-a_{i}\left(k+\delta_{q}\right)\right|+\left|\left[b_{i}\left(k+\delta_{q}\right)-b_{i}\left(k+\delta_{p}\right)\right]\left(x_{i}\left(k+\delta_{q}\right)\right)^{\theta_{i i}}\right|+\sum_{j=1, j \neq i}^{n}\left|\frac{c_{i j}\left(k+\delta_{p}\right)}{d_{i j}}\left[\left(x_{j}\left(k+\delta_{p}\right)\right)^{\theta_{i i}}-\left(x_{j}\left(k+\delta_{q}\right)\right)^{\theta_{i i}}\right]\right| \\
+\left|\left[e_{i}\left(k+\delta_{q}\right)-e_{i}\left(k+\delta_{p}\right)\right] u\left(k+\delta_{q}\right)\right|+\left|e_{i}\left(k+\delta_{p}\right)\right|\left|u\left(k+\delta_{q}\right)-u\left(k+\delta_{p}\right)\right| . \quad(4)
\end{gathered}
$$

Let $\varepsilon_{1}$ be an arbitrary positive number. By the almost periodicity of $\left\{a_{i}(k)\right\},\left\{b_{i}(k)\right\},\left\{c_{i j}(k)\right\}$ and $\left\{e_{i}(k)\right\}$ and the boundedness of $\left(x_{1}(k), x_{2}(k), \ldots, x_{n}(k), u_{1}(k), u_{2}(k), \ldots, u_{n}(k)\right)$, it follows from Theorem 2.3 that there exists a positive integer $K_{1}=K_{1}\left(\varepsilon_{1}\right)$ such that, for any $\delta_{q} \geq \delta_{p} \geq K_{1}$ and $k \in Z^{+}$(if necessary, we can choose subsequences of $\left\{\delta_{p}\right\}$ and $\left\{\delta_{q}\right\}$,

$$
\begin{gathered}
\left|a_{i}\left(k+\delta_{q}\right)-a_{i}\left(k+\delta_{p}\right)\right|<\frac{\varepsilon_{1}}{4},\left|\left[b_{i}\left(k+\delta_{q}\right)-b_{i}\left(k+\delta_{p}\right)\right]\left(x_{i}\left(k+\delta_{q}\right)\right)^{\theta_{i i}}\right|<\frac{\varepsilon_{1}}{4}, \\
\sum_{j=1, j \neq i}^{n}\left|c_{i j}\left(k+\delta_{q}\right)-c_{i j}\left(k+\delta_{p}\right)\right|<\frac{\varepsilon_{1}}{4},\left|\left[e_{i}\left(k+\delta_{q}\right)-e_{i}\left(k+\delta_{p}\right)\right] u_{i}\left(k+\delta_{q}\right)\right|<\frac{\varepsilon_{1}}{4} .
\end{gathered}
$$

It follows from (4.5)-(4.8) that, for $k \in Z^{+}$and $\delta_{q} \geq \delta_{p} \geq K_{1}$,

$$
\begin{aligned}
& \left|\ln x_{i}\left(k+1+\delta_{p}\right)-\ln x_{i}\left(k+1+\delta_{q}\right)\right| \\
& \leq \max \left\{\left|1-\theta_{i i} b_{i}^{l}\left(m_{i}-\varepsilon\right)^{\theta_{i j}}\right|,\left|1-\theta_{i i} b_{i}^{u}\left(M_{i}+\varepsilon\right)^{\theta_{i j}}\right|\right\},\left|\ln x_{i}\left(k+\delta_{p}\right)-\ln x_{i}\left(k+\delta_{q}\right)\right| \\
& +\sum_{j=1, j \neq i}^{n} \frac{\theta_{i j} c_{i j}^{u}\left(M_{j}+\varepsilon\right)^{\theta_{i j}}}{d_{i j}}\left|\ln x_{j}\left(k+\delta_{p}\right)-\ln x_{j}\left(k+\delta_{q}\right)\right|+\left|e_{i}\left(k+\delta_{p}\right)\right|\left|u_{i}\left(k+\delta_{p}\right)-u_{i}\left(k+\delta_{q}\right)\right| .
\end{aligned}
$$

Similar, we get

$$
\begin{aligned}
& \left|u_{i}\left(k+1+\delta_{p}\right)-u_{i}\left(k+1+\delta_{q}\right)\right| \\
& \quad \leq\left(1-f_{i}^{l}\right)\left|u_{i}\left(k+\delta_{p}\right)-u_{i}\left(k+\delta_{q}\right)\right|+\sum_{j=1}^{n} g_{i j}^{u} M_{j}\left|\ln x_{j}\left(k+\delta_{p}\right)-\ln x_{j}\left(k+\delta_{q}\right)\right|+\varepsilon_{1} .
\end{aligned}
$$

In view of $(\mathrm{H} 2)$, we can chose an $\varepsilon_{1}>0$ such that 


$$
\begin{aligned}
& \rho_{i}^{\varepsilon_{1}}=\max \left\{1-\theta_{i i} b_{i}^{l}\left(m_{i}-\varepsilon_{1}\right)^{\theta_{i j}}|,| 1-\theta_{i i} b_{i}^{u}\left(M_{i}+\varepsilon_{1}\right)^{\theta_{i j}} \mid\right\}+\sum_{j=1, j \neq i}^{n} \frac{\theta_{i j} c_{i j}^{u}\left(M_{j}+\varepsilon_{1}\right)^{\theta_{i j}}}{d_{i j}}+e_{i}^{u}+\varepsilon_{1}<1, \\
& \varphi_{i}^{\varepsilon_{1}}=1-f_{i}^{l}+\sum_{j=1}^{n} g_{i j}^{u}\left(M_{j}+\varepsilon_{1}\right)+\varepsilon_{1}<1, i=1,2, \cdots, n .
\end{aligned}
$$

Let $\rho_{i}=\max \left\{\rho_{i}^{\varepsilon_{1}}, \varphi_{i}^{\varepsilon_{1}}\right\}$. Then $0<\rho_{i}<1$. In view of (4.9) and (4.10), we get

$$
\begin{gathered}
\max \left\{\left|\ln x_{i}\left(k+1+\delta_{p}\right)-\ln x_{i}\left(k+1+\delta_{q}\right)\right|,\left|u_{i}\left(k+1+\delta_{p}\right)-u_{i}\left(k+1+\delta_{q}\right)\right|\right\} \\
\leq \rho_{i} \max \left\{\left|\ln x_{i}\left(k+\delta_{p}\right)-\ln x_{i}\left(k+\delta_{q}\right)\right|,\left|u_{i}\left(k+\delta_{p}\right)-u_{i}\left(k+\delta_{q}\right)\right|\right\} .
\end{gathered}
$$

For convenience, we introduce $\varphi_{i}\left(k, \delta_{p}, \delta_{q}\right)$ through Then

$\varphi_{i}\left(k, \delta_{p}, \delta_{q}\right)=\max \left\{\left|\ln x_{i}(k+\delta p)-\ln x_{i}(k+\delta q)\right|,\left|u_{i}\left(k+\delta_{p}\right)-u_{i}\left(k+\delta_{q}\right)\right|\right\}, k \in Z^{+}, \delta_{p}>0, \delta_{q}>0$.

$$
\begin{aligned}
\varphi_{i}\left(k, \delta_{p}, \delta_{q}\right)<\rho_{i} \max \left\{\varphi_{i}\left(k-1, \delta_{p}, \delta_{q}\right)\right\}+\varepsilon_{1}, \\
\varphi_{i}\left(k-1, \delta_{p}, \delta_{q}\right)<\rho_{i} \max \left\{\varphi_{i}\left(k-2, \delta_{p}, \delta_{q}\right)\right\}+\varepsilon_{1}, \\
\cdots \cdots \cdots \cdots, \\
\varphi_{i}\left(1, \delta_{p}, \delta_{q}\right)<\rho_{i} \max \left\{\varphi i\left(0, \delta_{p}, \delta_{q}\right)\right\}+\varepsilon_{1} .
\end{aligned}
$$

And we have

$$
\left.\varphi_{i}\left(k, \delta_{p}, \delta_{q}\right)<\rho_{i}^{k} \max \varphi_{i}^{\{}\left(0, \delta_{p}, \delta_{q}\right)\right\}+\frac{1-\rho_{i}^{k}}{1-\rho_{i}^{k}} \varepsilon_{1}
$$

for $\mathrm{k} \in \mathrm{Z}^{+}$and $\delta_{q} \geq \delta_{p} \geq K_{1}$. $\geq K$,

Since $\rho_{i}<1$, for arbitrary $\varepsilon>0$, there exists a positive integer $K=K(\varepsilon)>K_{1}$ such that for any $\delta_{q} \geq \delta_{p}$

fork $\in \mathrm{Z}^{+}$.

$$
\varphi_{i}\left(k, \delta_{p}, \delta_{q}\right)<\frac{\varepsilon}{\max _{1 \leq i \leq n}\left\{M_{i}, 1\right\}}
$$

This combined with (4.6) gives us

$$
\left|x_{i}\left(k+\delta_{p}\right)-x_{i}\left(k+\delta_{q}\right)\right|<\varepsilon,\left|u_{i}\left(k+\delta_{p}\right)-u_{i}\left(k+\delta_{q}\right)\right|<\varepsilon
$$

It follows from Theorem 2.3 that the sequences $\left\{x_{i}(k)\right\}$ and $\left\{u_{i}(k)\right\}(i=1,2, \ldots, n)$ are asymptotically almost periodic. Thus we can express $\left\{x_{i}(k)\right\}$ and $\left\{u_{i}(k)\right\}$ as

$$
x_{i}(k)=p_{i}(k)+q_{i}(k), u_{i}(k)=v_{i}(k)+w_{i}(k)
$$

where $\left\{p_{i}(k)\right\}$ and $\left\{v_{i}(k)\right\}$ are almost periodic in $\mathrm{k} \in \mathrm{Z}$ and $q_{i}(k) \rightarrow 0$ and $w_{i}(k) \rightarrow 0$ as $k \rightarrow \infty$. In the following we show that $\left\{\left(p_{1}(k), p_{2}(k), \ldots, p_{n}(k), v_{1}(k), v_{2}(k), \ldots, v_{n}(k)\right)\right\}$ is an almost periodic solution of system (1.1).

In the following we show that $\left\{\left(p_{1}(k), p_{2}(k), \ldots, p_{n}(k), v_{1}(k), v_{2}(k), \ldots, v_{n}(k)\right)\right\}$ is an almost periodic positive solution of system (1.1).

From the properties of an almost periodic sequence, there exists an integer valued sequence $\left\{\delta_{p}\right\}, \delta_{p}$ $\rightarrow+\infty$ as $p \rightarrow+\infty$, such that

asp $\rightarrow+\infty$.

$$
\begin{gathered}
a_{i}\left(k+\delta_{p}\right) \rightarrow a_{i}(k), b_{i}\left(k+\delta_{p}\right) \rightarrow b_{i}(k), c_{i j}\left(k+\delta_{p}\right) \rightarrow c_{i j}(k), \\
e_{i}\left(k+\delta_{p}\right) \rightarrow e_{i}(k), f_{i}\left(k+\delta_{p}\right) \rightarrow f_{i}(k), g_{i j}\left(k+\delta_{p}\right) \rightarrow g_{i j}(k),
\end{gathered}
$$

It is easy to know that $x_{i}\left(k+\delta_{p}\right) \rightarrow p_{i}(k), u_{i}\left(k+\delta_{p}\right) \rightarrow v_{i}(k)$ as $p \rightarrow \infty$, then we have

$$
\begin{aligned}
& p_{i}(k+1)=\lim _{p \rightarrow \infty} x_{i}\left(k+1+\delta_{p}\right)=p_{i}(k) \text { ex }\left\{a_{i}(k)-b_{i}(k)\left(p_{i}(k)\right)^{\theta_{i i}}+\sum_{j=1, j \neq i}^{n} \frac{c_{i j}(k)\left(x_{j}(k)\right)^{\theta_{i j}}}{d_{i j}+\left(x_{j}(k)\right)^{\theta_{i j}}}-e_{i}(k) v_{i}(k)\right\}, \\
& v_{i}(k+1)=\lim _{p \rightarrow \infty} u_{i}\left(k+1+\delta_{p}\right)=\left[1-f_{i}(k)\right] v_{i}(k)+\sum_{j=1}^{n} g_{i j}(k) p_{j}(k)
\end{aligned}
$$

This prove that $p(k)=\left\{\left(p_{1}(k), p_{2}(k), \ldots, p_{n}(k), v_{1}(k), v_{2}(k), \ldots, v_{n}(k)\right)\right\}$ satisfied system (1.1), and $p(k)$ is a positive almost periodic solution of system (1.1).

Now, we show that there is only one positive almost periodic solution of system (1.1). For any two positivealmost periodic solutions $\left(p_{1}(k), p_{2}(k), \ldots, p_{n}(k), v_{1}(k), v_{2}(k), \ldots, v_{n}(k)\right)$ and $\left(z_{1}(k), z_{2}(k), \ldots\right.$, 
$\left.z_{n}(k), l_{1}(k), l_{2}(k), \cdots, l_{n}(k)\right)$ of system (1.1), we claim that $p_{i}(k)=z_{i}(k), v_{i}(k)=l_{i}(k),(i=1,2, \ldots, n)$ for all $k \in \mathbf{Z}^{+}$. Otherwise there must be at least one positive integer $K^{*} \in \mathbf{Z}^{+}$such that $p_{i}\left(K^{*}\right) \neq z_{i}\left(K^{*}\right)$ or $v_{j}\left(K^{*}\right)$ $\neq l_{j}\left(K^{*}\right)$ for a certain positive integerior $j$, i.e.,

$$
\Omega_{1}=\left|p_{i}\left(K^{*}\right)-z_{i}\left(K^{*}\right)\right|>0, \Omega_{2}=\left|v_{j}\left(K^{*}\right)-l_{j}\left(K^{*}\right)\right|>0
$$

So we can easily know that

$$
\begin{aligned}
& \Omega_{1}=\left|\lim _{p \rightarrow+\infty} p_{i}\left(K^{*}+\delta_{p}\right)-\lim _{p \rightarrow+\infty} z_{i}\left(K^{*}+\delta_{p}\right)\right|=\lim _{k \rightarrow+\infty}\left|p_{i}(k)-z_{i}(k)\right|>0, \\
& \Omega_{2}=\left|\lim _{p \rightarrow+\infty} v_{j}\left(K^{*}+\delta_{p}\right)-\lim _{p \rightarrow+\infty} l_{j}\left(K^{*}+\delta_{p}\right)\right|=\lim _{k \rightarrow+\infty}\left|v_{j}(k)-l_{j}(k)\right|>0,
\end{aligned}
$$

which is a contradiction to (4.4). Thus $p_{i}(k)=z_{i}(k), v_{i}(k)=l_{i}(k)(i=1,2, \ldots, n)$ hold for $\forall k \in \mathbf{Z}^{+}$. Therefore, system (1.1) admits a unique almost periodic positive solution which is globally attractive. This completes the proof of Theorem 4.2.

\section{Example and numerical simulation}

In this section, we give the following example to check the feasibility of our result.

Example Consider the following almost periodic discrete Gilpin-Ayala mutualism system with feedback controls

$$
\begin{aligned}
& x_{1}(k+1)=x_{1}(k) \exp \left\{1.1-0.022 \sin (\sqrt{3} k)-(1.05+0.013 \sin (\sqrt{5} k))\left(x_{1}(k)\right)^{1 / 2}\right. \\
& \left.+\frac{(0.025-0.001 \cos (\sqrt{2} k)) x_{2}(k)}{0.2+x_{2}(k)}+\frac{(0.02+0.0015 \cos (\sqrt{3} k)) x_{3}(k)}{0.4+x_{3}(k)}-(0.025-0.002 \cos (\sqrt{3} k)) u_{1}(k)\right\}, \\
& x_{2}(k+1)=x_{2}(k) \exp \left\{1.15-0.025 \sin (\sqrt{2} k)-(1.085+0.015 \sin (\sqrt{3} k))\left(x_{2}(k)\right)^{1 / 2}\right. \\
& \left.+\frac{(0.025+0.003 \cos (\sqrt{5} k)) x_{1}(k)}{0.35+x_{1}(k)}+\frac{(0.025-0.002 \cos (\sqrt{2} k)) x_{3}(k)}{0.2+x_{3}(k)}-(0.025+0.004 \sin (\sqrt{2} k)) u_{2}(k)\right\}, \\
& x_{3}(k+1)=x_{3}(k) \exp \left\{1.25-0.03 \sin (\sqrt{5} k)-(1.1-0.024 \sin (\sqrt{2} k))\left(x_{3}(k)\right)^{1 / 2}\right. \\
& \left.+\frac{(0.03-0.002 \cos (\sqrt{2} k)) x_{1}(k)}{0.2+x_{1}(k)}+\frac{(0.028+0.0015 \cos (\sqrt{3} k)) x_{2}(k)}{0.25+x_{2}(k)}-(0.02+0.002 \cos (\sqrt{3} k)) u_{3}(k)\right\}, \\
& \Delta u_{1}(k)=-(0.93-0.03 \sin (\sqrt{2} k)) u_{1}(k)+(0.015+0.005 \sin (\sqrt{3} k)) x_{1}(k) \\
& +(0.013-0.004 \sin (\sqrt{3} k)) x_{2}(k)+(0.024-0.005 \cos (\sqrt{5} k)) x_{3}(k), \\
& \Delta u_{2}(k)=-(0.924-0.04 \sin (\sqrt{3} k)) u_{2}(k)+(0.018-0.004 \sin (\sqrt{5} k)) x_{1}(k) \\
& +(0.015-0.005 \cos (\sqrt{2} k)) x_{2}(k)+(0.014+0.004 \sin (\sqrt{2} k)) x_{3}(k), \\
& \Delta u_{3}(k)=-(0.936-0.035 \cos (\sqrt{5} k)) u_{3}(k)+(0.017-0.006 \cos (\sqrt{2} k)) x_{1}(k) \\
& +(0.013-0.005 \sin (\sqrt{3} k)) x_{2}(k)+(0.014+0.005 \cos (\sqrt{2} k)) x_{3}(k) .
\end{aligned}
$$

By simple computation, we derive

$$
\rho_{1} \approx 0.101, \rho_{2} \approx 0.211, \rho_{3} \approx 0.108, \varphi_{1} \approx 0.009, \varphi_{2} \approx 0.053, \varphi_{3} \approx 0.101 \text {. }
$$

It is easy to see that the conditions of Theorem 4.2 are verified. Therefore, system (5.1) has a unique positive almost periodic positive solution which is globally attractive. Our numerical simulations support our results (see Figure1). 

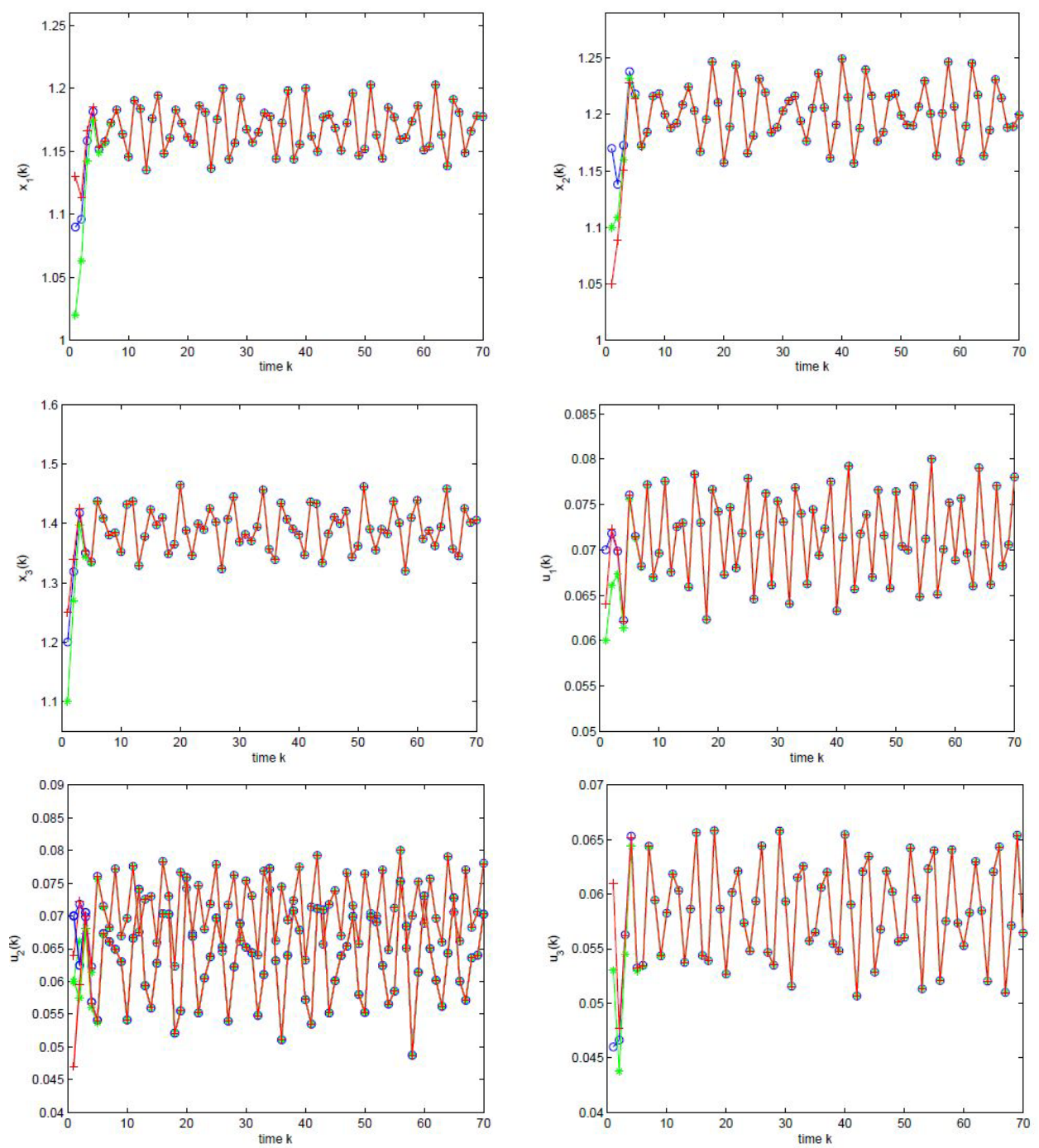

Figure 1. Dynamic behavior of positive almost periodic solution $\left(\mathrm{x}_{1}(\mathrm{k}), \mathrm{x}_{2}(\mathrm{k}), \mathrm{x}_{3}(\mathrm{k}), \mathrm{u}_{1}(\mathrm{k}), \mathrm{u}_{2}(\mathrm{k})\right.$, $\left.\mathrm{u}_{3}(\mathrm{k})\right)$ ofsystem (5.1) with the three initial conditions(1.09,1.17,1.2,0.07,0.07,0.046), (1.02,1.1,1.1,0.06,0.06,0.053) and(1.13,1.05,1.25,0.064,0.047,0.061) for $\mathrm{k} \in[1,70]$, respectively.

\section{Concluding remarks}

In this paper, assuming that the coefficients in system (1.1) are bounded non-negative almost periodic positive sequences, we obtain the sufficient conditions for the existence of a unique almost periodic positive solution which is globally attractive. By comparative analysis, we find that when the coefficients in system (1.1) are almost periodic, the existence of a unique almost periodic positive solution of system (1.1) is determined by the global attractivity of system (1.1), which implies that there is no additional condition to add.

Furthermore, for the almost periodic multispecies discrete Gilpin-Ayala mutualism system with feedback controls and timed elays, we would like to mention here the question of how to study the almost periodicity of the system and whether the existence of a unique almost periodic positive solution is determined by the global attractivity of the system or not. It is, in fact, a very challenging problem, and we leave it for our future work. 


\section{Acknowledgment}

There are no financial interest conflicts between the authors and the commercial identity.

\section{References}

1. Yijie Wang, Periodic and almost periodic solutions of a nonlinear single species discrete model with feedback control, Applied Mathematics and Computation, 219(2013)5480-5496.

2. Tianwei Zhang, XiaorongGan, Almost periodic solutions for a discrete fishing model with feedback control and time delays, Commun Nonlinear SciNumerSimulation, 19(2014)150-163.

3. Tianwei Zhang, Yongkun Li, Yuan Ye, Persistence and almost periodic solutions for a discrete fishing model with feedback control, Communications in Nonlinear Science and Numerical Simulation, 16(2011)1564-1573.

4. A.M. Fink, G. Seifert, Liapunov functions and almost periodic solutions for almost periodic systems, Journal of Differential Equations, 5(1969)307-313.

5. Rong Yuan, The existence of almost periodic solutions of retarded differential equations with piecewise constant argument, Nonlinear Analysis, 48(2002)1013-1032.

6. Hui Zhang, FengFeng, Bin Jing, Yingqi Li, Almost periodic solution of a multispecies discrete mutualism system with feedback controls, Discrete Dynamics in Nature and Society, 2015, Article ID 268378, 14 pages.

7. R.P. Agarwal, M. Bohner, P. Rehak, Half-linear dynamic equations, in: Nonlinear Analysis and Applications to V. Lakshmikantham on his 80th Birthday, vol.1, Kluwer Academic Publishers,Dordrecht, 2003, 1-57.

8. Yonghong Fan, LinlinWang, Permanence for a discrete model with feedback control and delay, Discrete Dynamics in Natureand Society, 2008, 8 pages. 